\title{
Sonographic Association Between Fatty Liver and Gall Baldder
} Stone

\author{
Habib-ur Rehman* \\ Medical Imaging Doctor, Department of Allied Health Sciences, Superior College Lahore, University Campus, \\ 17-KM Raiwaind Road, Kot Arain, Lahore,Pakistan \\ Pakeeza Jameel \\ Medical Imaging Doctor, Department of Allied Health Sciences, Superior College Lahore, University Campus, \\ 17-KM Raiwaind Road, Kot Arain, Lahore,Pakistan
}

Muhammad Sohaib Khalid

Medical Imaging Doctor, Department of Allied Health Sciences, Superior College Lahore, University Campus, 17-KM Raiwaind Road, Kot Arain, Lahore,Pakistan

Dr. Shafqat Rehman

Medical Imaging Doctor,Masters in Medical Imaging Technology, Lecturer, Department of Allied Health Sciences, Superior College Lahore, University Campus, 17-KM Raiwaind Road, Kot Arain, Lahore,Pakistan

Dr. Muhammad Uzair

Medical Imaging Doctor,Masters in Medical Imaging Technology, Lecturer, The University of Lahore, 1-KM Defence Road, near Bhuptian Chowk, Lahore, Pakistan

Rana Muhammad Athar Azeem Shams

Medical Imaging Doctor,Masters in Medical Imaging Technology, Lecturer, Department of Allied Health Sciences, Superior College Lahore, University Campus, 17-KM Raiwaind Road, Kot Arain, Lahore,Pakistan

Rana Muhammad Bakhtawar Khan Sajawal

Masters in Mathematics, Lecturer, Department of Allied Health Sciences, Superior College Lahore, University Campus, 17-KM Raiwaind Road, Kot Arain, Lahore,Pakistan

\section{The research is financed by Asian Development Bank. No. 2006-A171(Sponsoring information)}

\section{Abstract}

Background: Fatty liver (chronic liver disease) was most frequently found associated with gall stones. It arises due to accumulation of lipid in hepatocytes mainly triglyceride. Due to high existence of obesity in the population, the risk of fatty liver and gall stones also increases. In Pakistan the prevalence of fatty liver was 15$20 \%$. Fatty liver and gall stones could easily be observed on ultrasound.

Objective: To evaluate the sonographic association between fatty liver and gall bladder Stone.

Study Design: Descriptive Study was conducted

Settings: Sanabil health services. Lahore

Period: Four months

Material \& Methods: In our study patients presents with epigastric pain high cholesterol level and previous history of gall stones. While patient with the history of cholecystectomy were excluded. All the data had been composed from Sanabil Health Services Lahore. After informed consent, data was composed through ultrasound machine GE LOGIQ P7 with convex probe (frequency 2.5- 5MHZ)

Results: According to results total volume of patient was 220. Now we were comparing between cholelithiasis and grading of fatty liver. Total no of GRADE 1 fatty liver patients were 63 out of 220 in which 13 patients were showing absence of GS while 50 patients showed GS. Total no of GRADE II fatty liver patients were 89 out of 220 in which 36 patients were showing absence of GS while 53 patients showed GS. Total no of GRADE III fatty liver patients were 68 out of 250 in which 20 patients were showing absence of GS while 48 patients showed GS. Total no of 69 out of 220 patients showed absence of GS while 151 patients out of 220 showed with GS.

Conclusion: We concluded that the patients who had high cholesterol level, gall stones are associated with fatty liver disease. Moreover fatty liver disease was more common in females than males.

Keywords: Nonalcoholic fatty liver, chronic liver disease, Gallbladder, Gall stones, Ultrasound and cholecystokinin.

DOI: $10.7176 / \mathrm{JHMN} / 91-09$

Publication date:July $31^{\text {st }} 2021$ 


\section{Introduction}

Obesity, diabetes, hyperlipidemia, and hyperinsulinemia are also the predisposing factors for fatty liver and gallstones ${ }^{1,2,3}$.subsequently of reduced gallbladder motility and enlarged bile lysogenicity, patients with gallstones might be at risk of emerging fatty liver ${ }^{4}$. It occurs from the assemblage of lipid in liver cell mostly triglyceride ${ }^{5,6}$.This condition can turn into more worst like liver cirrhosis. In citizens, its prevalence is $15-40 \%$ and increasing around the world ${ }^{7}$. In community the prevalence of the fatty liver in South-Asia and the South East Asia it scale from 5 to $30 \%$. Lately a health center established studies in our country Pakistan has manifest $14 \%$ frequency of fatty liver. In India it differs from $5-28 \%$ in overall population. Indian have increased incidence of visceral fat accretion, which might present from childbirth ${ }^{8,9}$. While in other countries like Japan have $9-14 \%$ prevalence, Hong Kong have $16 \%$, Taiwan have $11-41 \%$ and Indonesia have $30 \%$ prevalence. In United States about $20 \%$ of populations have fatty liver ${ }^{10}$. It might be develop up to $80 \%$ of obese and up to $20 \%$ in normal weight people ${ }^{11}$. There are certain risky reasons associated with fatty liver disease like, obesity, high dietary intake, diabetes mellitus type II. Fatty acid metabolism problems are also accountable for pathogenesis of hepatic steatosis, which may be because of disproportion in energy intake and it's burning, resulting in lipid storage in the liver ${ }^{12}$. The additional liver bile-duct classification has been separated into four different topographic sections. Duct carrying bile and GB, right as well as left hepatic-ducts, common hepatic along with bile duct with its supra and retro-duodenal portions and the pancreatic and intra-duodenal portions ${ }^{13}$. Nonalcoholic hepatic steatosis raise to occurrence of liver fat disease when no additional etiologies of secondary liver fat collects (e.g., heavy alcohol intake, hypothyroidism, medications, etc.) are present. NAFLD is the most likely cause of cryptogenic cirrhosis, since it can lead towards cirrhosis or Hepatocellular-Carcinoma (HCC) ${ }^{14}$. Fatty liver diagnose on the base of the brilliance of liver and the appearance of disperse echogenicity inparenchyma of liver on abdomen ultrasound. Liver function test (LFTs) is not sufficient to authorize fatty liver ${ }^{15}$.

Intrahepatic fat and Gallbladder stones are both related to increase the cholesterol .With this study we want to find a relation between fatty liver and gallbladder stone, so that we can predicted the possibility of formation of gallbladder stone and reduce the risk of cholelithiasis in patients having fatty liver.

\section{Results}

The static of gender 220, grading of fatty liver,total valid in gender 220, GS 220,cholesterol 220, while Diabetic were 220.while missing is $0,0,0,0$ and 0 respectively. Mean of gender 25 , mean of grading of fatty liver 2.02 , mean of cholesterol 5.43, mean of GS is 8.69 while mean of diabetes is 2.38 .Mode of of gender 0 , mode of grading of fatty liver 2 , mode of cholesterol 5 , mode of GS is 9 while mode of diabetes is 4 . Minimum of gender is 0 and grading of fatty liver is 0 , cholesterol is $5, \mathrm{GS}$ is 8 and diabetes is 0 .Maximum of gender is 1 , grading of fatty liver is 3 , cholesterol is 6 , GS is 9 and diabetes is 4

\begin{tabular}{|c|c|c|c|c|c|c|c|}
\hline & & Age & Gender & $\begin{array}{l}\text { Grading of fatty } \\
\text { liver }\end{array}$ & Cholestrole level & Gall Stones & Diabetic \\
\hline \multirow[t]{2}{*}{$\mathrm{N}$} & Valid & 220 & 220 & 220 & 220 & 220 & 220 \\
\hline & Missing & 0 & 0 & 0 & 0 & 0 & 0 \\
\hline \multicolumn{2}{|c|}{ Mean } & 32.51 & .25 & 2.02 & 5.43 & 8.69 & 2.38 \\
\hline \multicolumn{2}{|c|}{ Mode } & 33 & 0 & 2 & 5 & 9 & 4 \\
\hline \multicolumn{2}{|c|}{ Minimum } & 20 & 0 & 1 & 5 & 8 & 0 \\
\hline \multicolumn{2}{|c|}{ Maximrgt5 um } & 54 & 1 & 3 & 6 & 9 & 4 \\
\hline
\end{tabular}

\section{Discussion}

In current study it was noted that, there was association between gall bladder stones and fatty liver disease. A study was accomplished at civil hospital Karachi in surgical unit 1. In this study, selective sample comprising 88 patients was involved of both genders with ultra-sonographic evidence of gallstone regardless of cholecystitis. Non alcoholic fatty liver disease was found in 62.5 percent $(\mathrm{n}=55)$ of the participants, while metabolic syndrome was found in 28.4 percent $(\mathrm{n}=25) .26 .94$ percent has a BMI of lesser than $18,32.12$ had a BMI of 18 to 25 , and 40.93 percent has a BMI of more than 25 . A family history of cholelithiasis was found in 46.6 percent of the participants. Gall Stones patients with non alcoholic fatty liver disease had a substantial p-value of 0.034 for having a first-degree relative with cholelithiasis, furthermore this was incidental in cases of metabolic disorder, with a P-value of 0.190 . This research discovered a connection between metabolic disorders, gallstones, and fatty liver disease. The gallstone in this association was more evident in first-degree relatives of gallstone victims who had associated fatty liver diseases. while in this study ,the frequency ( NAFLD ) patients were 220 . In which grade I grading of fatty liver is 63 , percent was 28.6 . Valid percent was 28.6 and C.f is 28.6 .In which grade II grading of fatty liver is 89 , percent was 40.5 . Valid percent was 40.5 and Cuff is 69.1 .while grade III grading of fatty liver is 68 , percent was 30.9 , valid percent was 30.9 and C.f is 100 . Total 220 patients showed 
100 percent C.f. while the gall stone total no of 220 patients. In which the absence of GS were 69 out of $220, \%$ is 31.4 , valid percent is 31.4 and c.f is 31.4 . While the presence of GS were 151 out of $220 \%$ is 68.6 , valid percent is 68.6 and c.f is 100 . Total 220 patients were 100 valid percentage.

A systematic review of studies was carried out in 2015 to survey the relation $\mathrm{b} / \mathrm{w}$ fatty liver and gallstone and its risk factors. Eight studies were involved which consists of 43,749 people from distinct ethnics and regions. Five trials were carried out in subgroups of gallbladder disease patients in the NAFLD population. NAFLD was found to be strongly linked to GD (odds-ratio [OR] $=1.75$, 95 percent confidence interval [CI]: 1.51-2.04, P 0.01). Age, BMI, fasting glucose, homeostasis model assessment-insulin resistance (HOMA-IR), and the demonstration of metabolic syndrome (MS), also women, all increased incidence of GD in NAFLD participants. They came to the conclusion that there is a connection between NAFLD and gallstone disease. Total volume of patient was 220. Now we were comparing between cholelithiasis and grading of fatty liver. Total no of GRADE 1 fatty liver patients were 63 out of 220 in which 13 patients were showing absence of GSD while 50 patients showed GSD. Total no of GRADE II fatty liver patients were 89 out of 220 in which 36 patients were showing absence of GSD while 53 patients showed GSD. Total no of GRADE III fatty liver patients were 68 out of 250 in which 20 patients were showing absence of GSD while 48 patients showed GS. Total no of 69 out of 220 patients showed absence of GSD while 151 patients out of 220 showed with GS. Fatty liver disease cannot be confirmed only on ultrasonography. In the sample size of 220 patients, most of them were females because trend of routine ultrasound examination was more common in females than males and incidence of gallstones disease was also higher in females.

\section{Conclusion}

We discovered that GB-stone are associated with fatty liver disease. Furthermore fatty liver disease is more common in females as compared to males.

\section{Conflict of Interest}

There is no conflict of interest in this research.

\section{Reference}

1. Fracanzani AL, Valenti L, Russello M, Miele L, Bertelli C, Bellia A, Masetti C, Cefalo C, Grieco A, Marchesini G, Fargion S. Gallstone disease is associated with more severe liver damage in patients with non-alcoholic fatty liver disease. PloS one. 2012 Jul 25;7(7):e41183. https://doi.org/10.1371/journal.pone.0041183

2. Loria P, Lonardo A, Lombardini S, Carulli L, Verrone A, Ganazzi D, Rudilosso A, D’AMICO RO, Bertolotti M, Carulli N. Gallstone disease in non-alcoholic fatty liver: prevalence and associated factors. Journal of gastroenterology and hepatology. 2005 Aug;20(8):1176-84. https://doi.org/10.1111/j.14401746.2005.03924.x

3. Kwak MS, Kim D, Chung GE, Kim W, Kim YJ, Yoon JH. Cholecystectomy is independently associated with nonalcoholic fatty liver disease in an Asian population. World Journal of Gastroenterology: WJG. 2015 May 28;21(20):6287.

4. Li X, Guo X, Ji H, Yu G, Gao P. Gallstones in patients with chronic liver diseases. BioMed research international. 2017 Jan 31;2017. https://doi.org/10.1155/2017/9749802

5. Browning JD, Horton JD. Molecular mediators of hepatic steatosis and liver injury. The Journal of clinical investigation. 2004 Jul 15;114(2):147-52.

6. Ahmed F, Baloch Q, Memon ZA, Ali I. An observational study on the association of nonalcoholic fatty liver disease and metabolic syndrome with gall stone disease requiring cholecystectomy. Annals of medicine and surgery. 2017 May 1;17:7-13. https://doi.org/10.1016/j.amsu.2017.03.015

7. Schuppan D, Gorrell MD, Klein T, Mark M, Afdhal NH. The challenge of developing novel pharmacological therapies for non-alcoholic steatohepatitis. Liver International. 2010 Jul;30(6):795-808. https://doi.org/10.1111/j.1478-3231.2010.02264.x

8. Amarapurkar DN, Hashimoto E, Lesmana LA, Sollano JD, Chen PJ, Goh KL, Asia-Pacific Working Party on NAFLD1. How common is non-alcoholic fatty liver disease in the Asia-Pacific region and are there local differences?. Journal of gastroenterology and hepatology. 2007 Jun;22(6):788-93. https://doi.org/10.1111/j.1440-1746.2007.05042.x

9. Raji A, Seely EW, Arky RA, Simonson DC. Body fat distribution and insulin resistance in healthy Asian Indians and Caucasians. The Journal of Clinical Endocrinology \& Metabolism. 2001 Nov 1;86(11):5366-71.

10. Rinella ME. Nonalcoholic fatty liver disease: a systematic review. Jama. 2015 Jun 9;313(22):2263-73..

11. Younossi Z, Anstee QM, Marietti M, Hardy T, Henry L, Eslam M, George J, Bugianesi E. Global burden of NAFLD and NASH: trends, predictions, risk factors and prevention. Nature reviews Gastroenterology \& hepatology. 2018 Jan;15(1):11-20.. 
12. Reddy JK, Sambasiva Rao M. Lipid metabolism and liver inflammation. II. Fatty liver disease and fatty acid oxidation. American Journal of Physiology-Gastrointestinal and Liver Physiology. 2006 May;290(5):G8528.https://doi.org/10.1152/ajpgi.00521.2005

13. Chen WJ, Ying DJ, Liu ZJ, He ZP. Analysis of the arterial supply of the extrahepatic bile ducts and its clinical significance. Clinical Anatomy: The Official Journal of the American Association of Clinical Anatomists and the British Association of Clinical Anatomists. 1999;12(4):245-9.

14. Qiao H.Q , MM , ZHU H.W,M.M . yu.X.y, houng.f.f and chen.y.Nonalcoholic fatty liver was associated with asymptomatic gall baldder stone in Chinese . Medicine ( baltimore ) 2017;96 (38)(6)

15. Liu J, Lin H, Zhang C, Wang L, Wu S, Zhang D, Tang F, Xue F, Liu Y. Non-alcoholic fatty liver disease associated with gallstones in females rather than males: a longitudinal cohort study in Chinese urban population. BMC gastroenterology. 2014 Dec;14(1):1-7. 